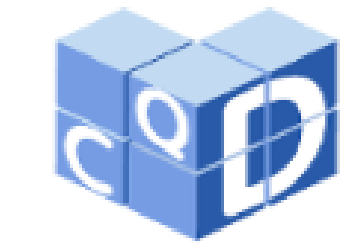

Revista Eletrônica

Paulista de Matemática

ISSN 2316-9664

Volume 10, dez. 2017

Edição Ermac

\section{Pedro Mantegazza}

Universidade de São Paulo EESC

pedro.mantegazza@usp.br

\section{Luben Cabezas-Gómez}

Universidade de São Paulo EESC

lubencg@sc.usp.br

\section{Felipe Costa Magazoni}

Universidade de São Paulo EESC

felipe.magazoni@gmail.com

\section{Modelagem de uma turbina radial para o ciclo de Rankine orgânico pelo método da linha média}

A radial inflow turbine modeling for the organic Rankine cycle by the mean line method

\section{Resumo}

A procura por novas fontes de energia útil tem suscitado o uso de fontes de baixo potencial térmico, como solar, geotérmica e térmica residual dos processos industriais. Nesse contexto, o uso de sistemas de geração de energia elétrica operando segundo o ciclo Rankine orgânico é bastante recomendado atualmente. Os fluidos orgânicos se caracterizam por baixo ponto de ebulição a altas pressões, baixo volume específico e possibilitam o uso de sistemas compactos. Entretanto, o uso de fontes de baixo potencial térmico resulta em uma eficiência energética baixa, sendo dispositivo de expansão de bastante importância. O expansor escolhido para o ciclo compreende uma turbina radial. $\mathrm{O}$ objetivo do trabalho é realizar o projeto preliminar de uma turbina radial de baixa potência para sistemas térmicos operando segundo um ciclo Rankine orgânico através de modelagem matemática e computacional, utilizando o método da linha média e considerando-se modelos de perdas presentes na literatura e cinco fluidos através do método de otimização do enxame de partículas. O fluido com melhor desempenho é o R123.

Palavras-chave: Ciclo Rankine orgânico. Turbina radial. Método da linha média. Modelagem matemática e Aplicações.

\begin{abstract}
The search for new useful energy sources has been stimulating the use of low thermal sources as solar, geothermal and residual thermal from industry processes. Electrical generation from organic Rankine cycle is then recommended in this context. The organic fluids are characterized by their low boiling point at high pressure, low specific volume and they allow more compact systems. However, low thermal energy sources imply in lower overall thermal efficiency, what emphasizes the importance of the expansion device. The choice in this work was an inward flow radial (IFR) turbine. The objective of this work is to develop the preliminary design of a low power IFR turbine for an organic Rankine cycle through mathematical and computational modeling by the use of the mean line method and loss models of the literature, considering five different fluids and an optimization procedure called particle swarm. The fluid with the best performance was R123.
\end{abstract}

Keywords: Organic Rankine cycle. Inward flow radial turbine. Mean line method. Mathematical modeling and applications. 


\section{Introdução}

A geração de energia elétrica a partir de fontes energéticas residuais e/ou renováveis, com sistemas térmicos operando segundo o ciclo Rankine orgânico (CRO) tem aumentado recentemente. O CRO emprega fluidos orgânicos, tais como hidrocarbonetos ou refrigerantes e opera a menores temperaturas e pressões. Com isso os riscos ligados à segurança e complexidade do sistema são reduzidos. Trabalhar com esses fluidos possibilita também seu uso com fontes de calor de baixo potencial energético, visto que eles possuem baixo ponto de ebulição, o que é bastante desejável no uso de energias renováveis e/ou residuais.

Algumas das características desejáveis para os fluidos CRO envolvem os seguintes fatores: inclinação positiva da curva de saturação do vapor (fluido seco), que permite a expansão do vapor sem que haja mudança de fase; baixo volume específico, o que diminui as dimensões do sistema; baixa viscosidade, para minimização das perdas por atrito; alta condutividade térmica, o que possibilita a obtenção de trocadores de calor menores; baixa reatividade com o ozônio (ODP - Ozone Depleting Potential) e baixo potencial estufa (PALTRINIERI, 2014).

No presente trabalho se estuda uma turbina radial a ser operada em baixa potência. Segundo Dixon e Hall (2010), esse modelo de turbina proporciona uma eficiência tão boa quanto das turbinas axiais, porém realizando um trabalho por estágio de expansão maior, além da facilidade de manufatura e robustez. Todavia, Whitfield e Baines (1991) afirmam que para menores potências uma turbina axial necessitaria de muitas pás de tamanho reduzido, aumentando a área em contato com o fluido de trabalho e consequentemente, as perdas por atrito e o fator de bloqueio causado pela camada limite. A folga entre as extremidades das pás e o envoltório da turbina provoca perdas por vazamento, que são bastante significativas em componentes axiais de dimensões reduzidas.

O objetivo deste artigo é apresentar um modelo para projeto preliminar de turbinas radiais com entrada radial empregando um procedimento similar ao apresentado em Rahbar et al. (2014) e uma metodologia de otimização de eficiência baseada nas variáveis de entrada do projeto de turbinas radiais. Esses autores apresentam uma metodologia de projeto de uma turbina radial baseado no método da linha média (MLM) e na otimização de parâmetros de entrada da turbina para operar num ciclo de Rankine orgânico. Os autores consideraram vários modelos de perdas da literatura relativos aos componentes da turbina estudada. No presente trabalho se consideram alguns modelos de perdas diferentes, obtendo-se uma ferramenta de projeto preliminar de turbinas radiais. O trabalho foi apresentado parcialmente no Encontro Regional de Matemática Aplicada e Computacional (ERMAC-2017) (MANTEGAZZA, CABEZAS-GÓMEZ, MAGAZONI, 2017). No presente artigo foi aumentada a seção que trata sobre a explicação da metodologia de modelagem e solução numérica e se apresentam resultados adicionais.

\section{Metodologia}

\subsection{O ciclo de Rankine orgânico}

Sistemas térmicos de geração de potência operando segundo o ciclo de Rankine formam uma das aplicações mais utilizadas para geração de energia elétrica a partir de fontes de energia operando em faixas de pressão constante. O ciclo Rankine simples consiste basicamente na realização dos quatros processo termodinâmicos mostrados na Figura 1. $\mathrm{O}$ fluido é bombeado do estado líquido 1 até o estado líquido 2 de alta pressão, que representa a entrada no trocador de calor denominado de evaporador. Na prática caldeiras de vapor são 
empregadas para transferir a energia térmica ao fluido de trabalho e produzir o vapor superaquecido representado pelo estado 3. Nesse estado, o vapor entra em uma turbina, aonde ocorre a expansão do fluido até o estado 4, caracterizada pela correspondente queda de pressão do fluido de trabalho e geração de trabalho mecânico no seu eixo. O processo subsequente é o resfriamento do fluido até o estado $1 \mathrm{em}$ um condensador à pressão constante, resultando no fechamento do ciclo mostrado na Figura 1. O fluido convencional utilizado no ciclo de Rankine é o vapor de'água, dada a sua disponibilidade e possibilidade de operação em diferentes níveis de pressão. No caso da água é necessário o aquecimento a altas temperaturas para que o potencial energético das fontes tradicionais de calor seja aproveitado de forma que sua relação de custo-benefício seja a maior possível.

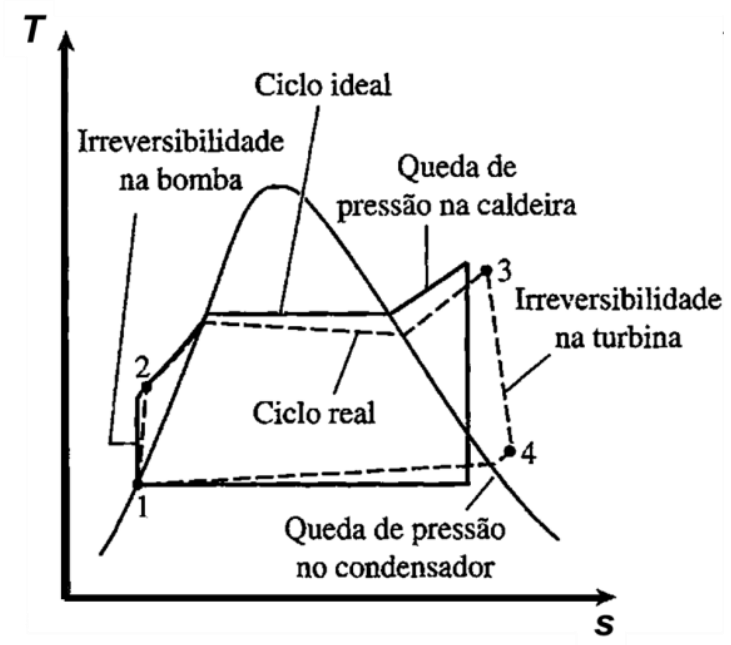

Figura 1 - Ciclo de Rankine real Fonte: (ÇENGEL; BOLES, 2013).

O ciclo de Rankine orgânico tem ganhado significado pelo uso de fluidos orgânicos, como fluidos refrigerantes, que possuem menor ponto de ebulição e menor volume específico, proporcionando um aproveitamento de fontes de calor de baixa temperatura em sistemas mais compactos. A menor temperatura de operação possibilita o uso de sistemas com materiais menos nobres e o ciclo é adequado para a utilização de fontes de energia renováveis, como solar, geotérmica, e de reaproveitamento energético, i.e., queima de biomassa, incineração de lixo ou reaproveitamento de gases oriundos da queima de combustíveis fósseis. Neste artigo se analisa o uso do ciclo CRO, considerando o uso de fluidos orgânicos e assumindo-se que não houve perdas termodinâmicas (quedas de pressão e diferenças de temperaturas com as respectivas fontes quente e fria) na passagem do fluido pela bomba, caldeira e condensador, mas para o sistema de geração, considerou-se uma eficiência de $96 \%$ para o conjunto de mecânico de transmissão do gerador elétrico e $96 \%$ também para a eficiência do gerador elétrico.

\subsection{Turbinas radiais e o método da linha média}

Turbinas radiais são componentes complexos de um sistema de geração de energia, responsáveis pela expansão do fluido em seus componentes e consequente conversão da energia térmica do fluido de trabalho em energia mecânica e elétrica, sucessivamente. Uma representação esquemática da geometria da turbina é apresentada na Figura 2. A turbina é 
composta por uma voluta (ponto 1) representando a entrada do expansor, cuja geometria descreve uma espiral, para que o escoamento, linear na tubulação de entrada, ganhe uma componente radial, além de uma redução de área ao longo do caminho, causando aceleração do fluido; um estator (entrada: ponto 2; saída: ponto 3), cuja redução da área de passagem provoca aumento da componente meridional da velocidade, juntamente com a redução do raio provocando aumento da velocidade tangencial com pás direcionadoras sem curvatura (e portanto, o ângulo da resultante da velocidade em relação à direção radial na entrada é igual ao da saída) em seu interior, para que o padrão escoamento seja homogeneizado na direção desejada na saída do estator; um rotor (entrada: ponto 4; saída: ponto 5), componente responsável pela conversão da energia cinética do fluido que realiza trabalho no eixo do gerador, além de provocar a mudança da direção do escoamento da direção radial para a axial. As dimensões da turbina estão expressas na Figura 1, as dimensões $b_{2}$ e $b_{3}$ são consideradas iguais a $b_{4}$.

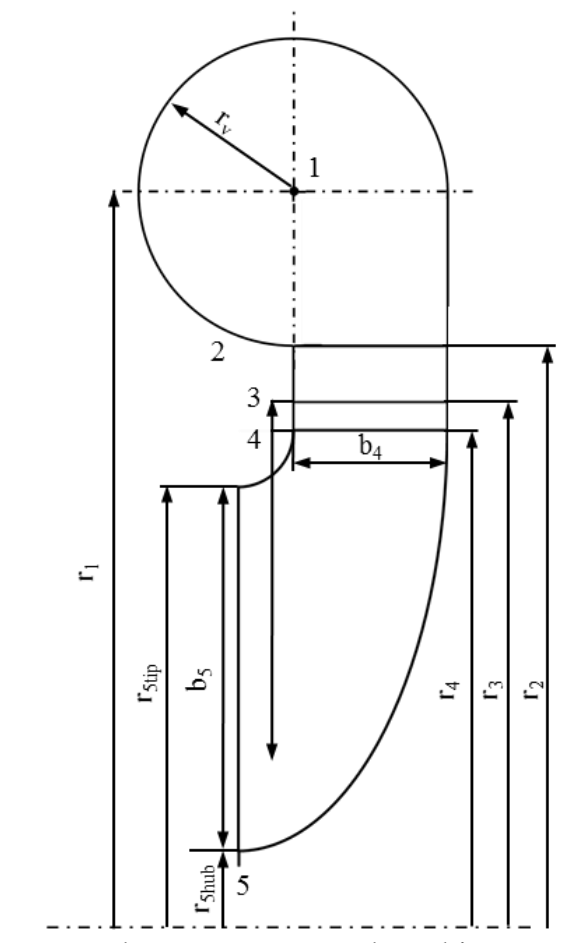

Figura 2 - Esquema dos componentes da turbina e a numeração dos estados Fonte: (RAHBAR et al., 2014).

A modelagem do escoamento no interior de uma turbina radial é realizada a partir do chamado método da linha média (MLM). O MLM consiste no uso de um modelo unidimensional, aproximando o escoamento na turbina baseada nas dimensões médias do estágio em consideração, obtendo-se dados preliminares da geometria dos componentes (WEI, 2014). Ao longo da linha média considerada em seções chave da turbina, como a fronteira entre seus componentes, são avaliados os estados termodinâmicos, assim como as variáveis fluidodinâmicas do escoamento, obtendo-se razoável precisão a partir de modelos de perdas hidrodinâmicas e termodinâmicas, apresentados na literatura. Entretanto, a geometria 3D das pás do rotor da turbina não é considerada, tendo em vista que essa análise mais aprofundada representa uma etapa posterior à apresentada neste trabalho, na qual o uso de ferramentas computacionais através de desenhos em CAD e uso da dinâmica dos fluidos computacional (DFC) possibilitam um refinamento do projeto e otimização dos componentes da turbina para que efeitos de perdas sejam minimizados, por exemplo. 
O MLM se baseia na aplicação dos conceitos físicos das leis de conservação: primeira e segunda leis da termodinâmica, que dizem respeito aos processos de mudança de estado do fluido no interior do expansor e servem como ferramenta para a definição de propriedades físicas do fluido de trabalho em cada seção analisada na turbina; a equação da continuidade, conservação da quantidade de movimento linear e angular, relacionadas com o comportamento dinâmico do fluido de trabalho no sistema, auxiliando no cálculo das perdas ocorridas no escoamento.

A velocidade do escoamento no interior da turbina pode ser decomposta em três componentes: radial, axial e tangencial. Uma simplificação nesse sistema de coordenadas permite considerar que a resultante das componentes radial e axial, denominada meridional, acompanhe a curvatura da linha de corrente do escoamento, possibilitando uma análise bidimensional das componentes de velocidade atuantes no sistema. A presença de um componente rotativo leva à necessidade do uso de componentes relativas da velocidade, considerando-se o referencial do rotor. A Figura 3 apresenta a convenção utilizada para a análise dos triângulos de velocidade. As direções indicadas na figura são os sentidos convencionados como positivo nesse trabalho.

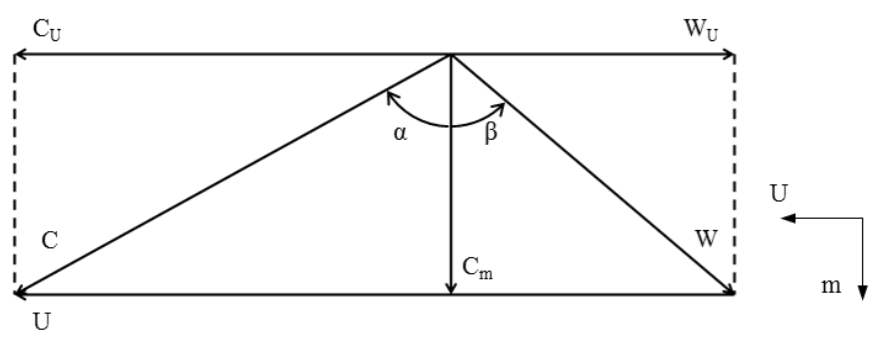

Figura 3 - Triângulo de velocidades.

A análise termodinâmica da turbina é realizada em conjunto com a análise de velocidades, dado que a entalpia de estagnação é igual à entalpia estática somada da energia cinética do fluido de trabalho, relacionada diretamente com a componente resultante da velocidade absoluta do escoamento. A partir de dados iniciais considerados realiza-se um projeto preliminar do rotor, cujos dados de saída são as dimensões principais do rotor. Essa análise baseia-se no diagrama h-s na Figura 4. Os pontos relativos aos estados respeitam a Figura 1. Seguindo a primeira lei da termodinâmica, observa-se que a entalpia de estagnação é mantida até o estado 4, pois, além de não haver geração de trabalho até esse ponto, admite-se que os processos ocorridos são adiabáticos, enquanto que entre os estados 4 e 5 há geração de trabalho, e com isso, uma redução na entalpia de estagnação. Uma expansão isentrópica representaria a condição de maior geração de trabalho no estágio da turbina e é representada pela diferença de entalpia entre os estados t1 e 5s. Essa é uma condição impossível de se obter considerando-se que o fluido deve sair da turbina com velocidade para que ele continue seu processo ao longo de todo o ciclo. Dada essa condição, que nunca será atingida, a entalpia de estagnação na saída será sempre maior do que a entalpia estática, nunca igual e, portanto, a diferença real de entalpia na entrada e na saída da turbina será igual à diferença de entalpia relativa aos estados t1 e t5. O produto entre essa diferença de entalpia e a vazão mássica é igual ao trabalho líquido entregue pela turbina. A eficiência térmica da turbina é o resultado da divisão entre a diferença de entalpia real e a entalpia do processo isentrópico. 


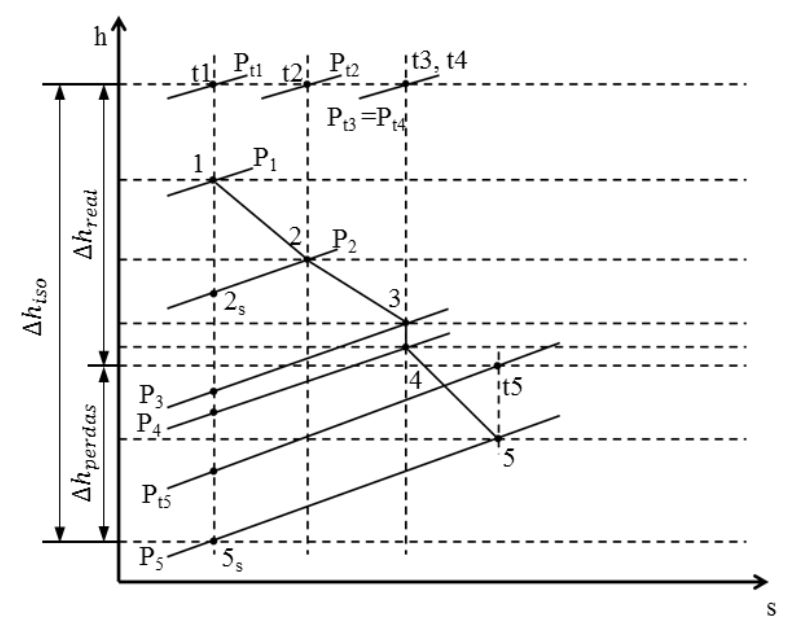

Figura 4 - Diagrama termodinâmico da turbina Fonte: Rahbar et al. (2014).

Inicialmente em um projeto é necessário usar a similaridade para que uma máquina já existente seja transformada em parâmetros adimensionais com o intuito de utilizá-los em novas aplicações (MOUSTAPHA et al., 2003). Além disso, a análise dimensional proporciona uma poderosa ferramenta no projeto de máquinas, que é a possibilidade de criação de modelos em escala a partir da obtenção de parâmetros adimensionais geométricos e cinemáticos (ÇENGEL; CIMBALA, 2015). Por exemplo, em vários casos de projetos de aplicações de turbinas radiais utiliza-se como fluido de trabalho hidrocarbonetos e gases provenientes de combustão. Para que haja uma primeira validação de um modelo em escala, sem que um protótipo trabalhe com esses gases, utiliza-se ar atmosférico como fluido de trabalho segundo Moustapha et al. (2003). Expandindo essa ideia para o ciclo de Rankine orgânico, tais parâmetros são: coeficiente de escoamento, coeficiente de carga das pás, coeficiente de potência, número de Reynolds, entre outros (MOUSTAPHA et al., 2003). Neste trabalho, os coeficientes adimensionais utilizados como dados de entrada do problema foram: o coeficiente de carga, que relaciona o trabalho gerado no rotor com suas dimensões e o coeficiente de fluxo, que relaciona a vazão volumétrica na turbina com suas dimensões.

O processo de cálculo se inicia com a definição dos parâmetros de entrada. Um programa foi escrito em MATLAB, por se tratar de um procedimento iterativo, e as propriedades termodinâmicas utilizadas foram calculadas com uma extensão do software Refprop para o MATLAB. Os cálculos das perdas encontrados na literatura são realizados normalmente a partir de correlações empíricas e estão expressas em termos de perda de entalpia e de pressão. Em cada componente da turbina ocorrem diferentes formas de perda. São elas: perdas na voluta, perdas no bocal, perdas no rotor pela folga de topo das pás, por escoamentos secundários e atrito e as perdas na saída.

O primeiro coeficiente de perdas é chamado de coeficiente de vórtice, utilizado em componentes que não possuem pás (MOUSTAPHA et al., 2003) e neste caso se utiliza na voluta. O coeficiente de vórtice é o único coeficiente de perdas apresentado que considera a equação da conservação do momento angular (Equação (1)) e que se relaciona com a perda de velocidade tangencial na voluta em relação à velocidade de entrada devido ao atrito com as paredes desse componente e sua consequente geração de vórtices.

$$
S C=\left(C_{1} r_{1}\right) /\left(C_{U 2} r_{2}\right)
$$


O cálculo da perda entálpica $\Delta h_{v o l}$ na voluta leva em consideração a perda de pressão na passagem do fluido, segundo Rahbar et al. (2014), e depende da velocidade do escoamento na saída da voluta. $\mathrm{O}$ coeficiente $k_{v o l}$, de perdas da voluta tem valor de 0,1 sugerido por Moustapha et al. (2003).

$$
\Delta h_{v o l}=k_{v o l} c_{2}^{2} / 2
$$

As perdas ocorridas no estator levam em conta os efeitos de atrito entre a superfície desse componente e o fluido, e o gradiente de pressão atuante no bordo de fuga das pás do estator. Segundo Rahbar et al. (2014) a perda entálpica por atrito nas pás do estator

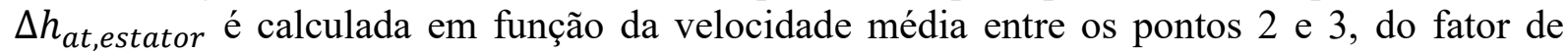
atrito do estator (Equação (4) e dependente do número de Reynolds do estator, Equação (3)), do diâmetro hidráulico (Equação (5)) e comprimento hidráulico do estator (Equação (6)) (RAHBAR et al., 2014).

$$
\begin{aligned}
& \overline{R e_{\text {boc }}}=\left[\left(C_{2} r_{2} \rho_{2} / \mu_{2}\right)+\left(C_{3} r_{3} \rho_{3} / \mu_{3}\right)\right] / 2 \\
& f_{\text {estator }}=8\left[\left(\left[\frac{8}{\overline{\text { Restator }_{\text {es }}}}\right)^{12}+\right.\right. \\
& d_{\text {hid,estator }}=\frac{b_{2} \cos \alpha_{2}}{\left(1+\frac{b_{2}}{\sigma_{\text {estator }}}\right)}+\frac{b_{3} \cos \alpha_{3}}{\left(1+\frac{b_{3}}{\sigma_{\text {estator }}}\right)} \\
& \left.\left.l_{\text {hid,estator }}=r_{2}-r_{3}+\left[\frac{37530}{\overline{\text { Re estator }}}\right]^{16}\right)^{-1,5}\right]^{\frac{1}{12}} \\
& \Delta h_{\text {at,estator }}=4 f_{\text {estator }}{\overline{C_{2,3}}}^{2} \frac{l_{\text {hid,estator }}}{d_{\text {hid,estator }}}
\end{aligned}
$$

A geometria das pás do estator é definida pela corda do estator, calculada através da Equação (8), passo entre as pás do estator (Equação (9), dependente da solidez do estator, valor de 1,35 sugerido por Glassman (1976) e número de pás do estator, calculado através da Equação (10).

$$
\begin{aligned}
& c o_{\text {estator }}=\sqrt{\left(r_{3} \cos \alpha_{3}\right)^{2}-\left({\left.r_{3}{ }^{2}-r_{2}{ }^{2}\right)}_{1}-r_{3} \cos \alpha_{3}\right.} \\
& p_{\text {estator }}=\left(\frac{c o_{\text {estator }}}{\sigma_{\text {estator }}}\right) \\
& Z_{\text {estator }}=\frac{2 \pi r_{3}}{p_{\text {estator }}}
\end{aligned}
$$

As perdas pelo bordo de fuga no estator se relacionam com o perfil de asa das pás desse componente, as quais possuem um gradiente de pressão responsável por uma diferença de velocidades entre seus dois lados. O lado de maior velocidade possui menor pressão, enquanto que o lado de menos velocidade possui maior pressão, causando um fluxo da região de menor pressão para a região de maior pressão, gerando vórtices e perdas. É assumido que o bordo de fuga possui espessura de $5 \%$ da pá do estator $b_{2}$ (Equação (11)) e assim, calcula-se a 
perda de pressão relativa ao bordo de fuga do estator (Equação (12), GLASSMAN, 1995) e

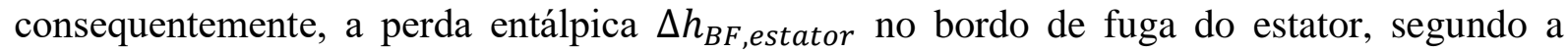
Equação (13) (WEI, 2014).

$$
\begin{aligned}
& t_{B F, \text { estator }}=0,05 b_{2} \\
& \Delta P_{B F, \text { estator }}=\frac{\rho_{3} c_{3}{ }^{2}}{2}\left(\frac{Z_{\text {bocal }} t_{B F, \text { estator }}}{2 \pi r_{3} \cos \alpha_{3}}\right)^{2} \\
& \Delta h_{B F, \text { estator }}=\frac{2}{\gamma M a_{3}{ }^{2}} \frac{\Delta P_{B F, \text { estator }}}{P_{3}\left(1+\frac{c_{3}{ }^{2}}{2 T_{3} C_{P 3}}\right)^{\frac{\gamma}{\gamma+1}}}
\end{aligned}
$$

Os mecanismos de perdas do rotor apresentam grande complexidade, dada a variedade de fenômenos ocorrendo simultaneamente devido ao movimento do fluido entre as pás do rotor.

O cálculo da perda entálpica da folga de topo das pás do rotor $\Delta h_{f o l, t o p}$ apresentada por Rahbar et al. (2014) considera a folga radial $\varepsilon_{r}$ e axial $\varepsilon_{x}$ (ambas expressas pela Equação (14)) como fração da altura da pá na saída do rotor $b_{5}$ e a causa das perdas é o vazamento de fluido através da folga entre o topo da pá e o envoltório da turbina. No cálculo da perda entálpica são levados em consideração coeficientes adimensionais de perdas axial $C_{x}$ e radial $C_{r}$, calculados conforme as Equações (15) e (16) respectivamente.

$$
\begin{aligned}
& \varepsilon_{x}=\varepsilon_{r}=0,04 b_{5} \\
& C_{x}=\frac{\left[1-\left(\frac{r_{5 t i p}}{r_{4}}\right)\right]}{\left[C_{m 4} b_{4}\right]} \\
& C_{r}=\frac{\left[1-\left(\frac{r_{5 t i p}}{r_{4}}\right)\right]}{\left[C_{m 4} b_{4}\right]} \\
& \Delta h_{\text {fol,topo }}=\left(\frac{U_{4}{ }^{3} Z_{\text {rotor }}}{8 \pi}\right)\left(0,4 \varepsilon_{x} C_{x}+0,75 \varepsilon_{r} C_{r}-0,3 \sqrt{\varepsilon_{x} \varepsilon_{r} C_{x} C_{r}}\right)
\end{aligned}
$$

O atrito causado pela rugosidade da pá é responsável por parte significativa das perdas no rotor, assim como as perdas causadas por escoamentos secundários. Este segundo mecanismo de perda possui como causa a recirculação do fluido devido a efeitos de turbulência no rotor. Wei (2014) apresenta o cálculo dessas duas perdas entálpicas como perdas na passagem segundo as Equações (25) e (26), considerando a largura média da garganta do rotor $o_{g}$ conforme Equação (18) e o coeficiente de separação e de escoamentos secundários no rotor $k_{\text {sep,sec }}$, conforme Equação (19). Nesse cálculo o diâmetro hidráulico (Equação (22)) e comprimento hidráulico do rotor (Equação (23)) utilizados são os mesmos propostos pelos cálculos de Rahbar et al. (2014), e a corda da pá é calculada segundo Ventura et al. (2012) através da Equação (24).

$$
\begin{aligned}
& o_{g}=\frac{\left(2 \pi r_{5} c_{m 5}\right)}{\left(Z_{\text {rotor }} w_{5}\right)} \\
& k_{\text {sep,sec }}=\frac{\left(r_{4}-r_{5}\right)}{o_{g}}
\end{aligned}
$$




$$
\begin{aligned}
& m_{f}=1 \text { se } k_{\text {sep }, \text { sec }} \geq 0,2 \\
& m_{f}=2 \text { se } k_{\text {sep,sec }}<0,2 \\
& d_{\text {hid,rotor }}=0,5\left(\frac{\left[4 \pi r_{4} b_{4}\right]}{\left[2 \pi r_{4}+Z_{\text {rotor }} b_{4}\right]}+\frac{\left[2 \pi\left(r_{5 \text { topo }}{ }^{2}-r_{5 \text { cubo }}{ }^{2}\right)\right]}{\left[\pi b_{5}+Z_{\text {rotor }} b_{5}\right]}\right) \\
& l_{\text {hid,rotor }}=\left(\frac{\pi}{2}\right)\left[\sqrt{\left.\frac{\left[\left(r_{4}-r_{5 t o p o}+\frac{b_{4}}{2}\right)^{2}+\left[\frac{\left(r_{5 t o p o}-r_{5 \text { cubo }}\right)}{2}\right]^{2}\right]}{2}\right]}\right. \\
& \mathrm{CO}_{\text {rotor }}=\frac{l_{\text {hid,rotor }}}{\pi \sqrt{2}} \\
& \Delta h_{\text {at,rotor }}=0,11 m_{f}\left(\frac{l_{\text {hid,rotor }}}{d_{\text {hid, }, \text { otor }}}\right) \frac{\left(w_{4}^{2}+\left(0,7 w_{5}\right)^{2}\right)}{2} \\
& \Delta h_{\text {sec,rotor }}=0,11 m_{f}\left[0,68\left(1-\left[\frac{r_{5}}{r_{4}}\right]^{2}\right) c o_{\text {rotor }} \frac{\cos \left(0,8 \beta_{5}\right)}{o_{g}}\right] \frac{\left(w_{4}{ }^{2}+\left(0,7 w_{5}\right)^{2}\right)}{2}
\end{aligned}
$$

As perdas pelo bordo de fuga no rotor seguem o mesmo procedimento proposto para o estator, com a diferença da porcentagem utilizada para a espessura do bordo de fuga na pá do rotor, sendo essa considerada como $4 \%$ da altura da pá do rotor na saída $b_{5}$, conforme Equação (27). O valor $M a_{r e l, 5}$ representa o número de Mach da velocidade relativa $W_{5}$, na saída do rotor.

$$
\begin{aligned}
& t_{B F, \text { rotor }}=0,04 b_{5} \\
& \Delta P_{B F, \text { rotor }}=\frac{\rho_{5} w_{5}{ }^{2}}{2}\left(\frac{Z_{\text {rotor }} t_{B F, \text { rotor }}}{2 \pi r_{5} \cos \beta_{5}}\right)^{2} \\
& \Delta h_{B F, \text { rotor }}=\frac{2}{\gamma M a_{\text {rel }, 5}{ }^{2}} \frac{\Delta P_{B F, \text { rotor }}}{P_{5}\left(1+\frac{c_{5}{ }^{2}}{2 T_{5} C_{P 5}}\right)^{\frac{\gamma}{\gamma+1}}}
\end{aligned}
$$

O modelo de perdas por incidência apresentado por Whitfield e Baines (1991) leva em consideração a existência de um ângulo ótimo de escoamento do fluido no rotor em relação às pás $\left(\beta_{\text {4ótimo }}\right.$, Equação (30)), sendo esse o ângulo relativo às perdas nulas por incidência. $\mathrm{O}$ modelo de perda entálpica por incidência $\Delta h_{\text {inc }}$ leva em consideração o desvio do ângulo real do escoamento em relação ao ângulo ótimo (Equação (31)). Uma vantagem do sistema CRO é o uso de ângulos de entrada mais agudos no rotor, dado que a temperatura de operação é mais baixa e consequentemente possuindo o material do rotor maior resistência mecânica, ele pode estar sujeito à maiores esforços de flexão, mais presentes nesse tipo de construção.

$$
\beta_{4 o ́ t i m o}=\tan ^{-1}\left[\frac{-1,98 \tan \left(\alpha_{4}\right)}{Z_{\text {rotor }}\left(1-\frac{1,98}{Z_{\text {rotor }}}\right)}\right]
$$

MANTEGAZZA, P.; CABEZAS-GÓMEZ, L.; MAGAZONI, F. C. Modelagem de uma turbina radial para o ciclo de Rankine orgânico pelo método da linha média. C.Q.D.- Revista Eletrônica Paulista de Matemática, Bauru, v. 10, p. 50-67, dez. 2017. Edição Ermac. 


$$
\Delta h_{\text {inc }}=\frac{1}{2} W_{4}^{2} \sin ^{2}\left(\beta_{4}-\beta_{4 o ́ t i m o}\right)
$$

O mecanismo de perdas por atrito no disco do rotor é causado pela presença de fluido entre o disco do rotor - a área atrás das pás - e a carcaça, sendo uma região de pequena folga entre os componentes e sujeita a efeitos de atrito do fluido que provocam um torque de desaceleração no rotor. Essa folga é descrita como $4 \%$ da largura da entrada do rotor $\left(b_{4}\right.$, Equação (32)) e o número de Reynolds é avaliado com a média das propriedades do fluido na entrada e saída do rotor (Equação (33)) (WEI, 2014). O coeficiente de atrito do cisalhamento do fluido é avaliado para o caso de escoamento laminar (Equação (34)) e turbulento (Equação (35)) e a perda entálpica por atrito no disco é calculada segundo a Equação (36) (WHITFIELD; BAINES, 1991).

$$
\begin{aligned}
& \varepsilon_{b}=0,05 b_{4} \\
& \overline{R e_{\text {rotor }}}=\frac{\bar{\rho}_{4,5} \bar{c}_{4,5} r_{4}}{\bar{\mu}_{4,5}} \\
& K_{f}=3,7 \frac{\left(\frac{\varepsilon_{b}}{r_{4}}\right)^{0,1}}{{\overline{R e} \text { rotor }^{0,5}}} \text { se } \overline{\operatorname{Re}_{\text {rotor }}}<10^{5} \\
& K_{f}=0,102 \frac{\left(\frac{\varepsilon_{b}}{r_{4}}\right)^{0,1}}{{\overline{R e_{\text {rotor }}}}^{0,2}} \text { se } \overline{\operatorname{Re}_{\text {rotor }}}>10^{5} \\
& \Delta h_{\text {at, disco }}=\frac{K_{f} \bar{\rho} U_{4}{ }^{3} r_{4}{ }^{2}}{4 \dot{m}}
\end{aligned}
$$

Por fim, o cálculo das perdas na saída é apresentado por Rahbar et al. (2014) como a energia cinética não aproveitada na conversão de energia cinética do fluido em trabalho no eixo do rotor (Equação (37)).

$$
\Delta h_{\text {ecin,rotor }}=\frac{c_{5}^{2}}{2}
$$

A metodologia de cálculo segue a proposta por Rahbar et al. (2014), com algumas modificações, conforme as citações mostradas junto das equações apresentadas anteriormente. Ela se baseia nos seguintes valores de entrada fixados (e seus intervalos): temperatura $\left(T_{t 1}=\right.$ $400-500 \mathrm{~K})$ e pressão de estagnação na entrada $\left(P_{t 1}=200-3500 \mathrm{kPa}\right)$, razão de pressão de estagnação para estática entre a entrada e saída $\left(E R_{t s}=2-15\right)$, respectivamente, rotação $(\omega=20000-70000 \mathrm{rpm})$, coeficiente de carga $(\Psi=0,8-2,4)$, coeficiente de fluxo $(\varphi=0,2-0,5)$, ângulo do vetor velocidade absoluta em relação à linha meridional na saída do rotor $\left(\alpha_{5}=0^{\circ}\right)$, razão entre o raio da raiz da pá na saída e o raio da entrada do rotor $\left(r_{5 h u b} / r_{4}=0,2\right)$, razão entre os raios de entrada e saída do estator $\left(r_{2} / r_{3}=1,2\right)$ e vazão mássica ( $\dot{m}=0,2-1,8 \mathrm{~kg} / \mathrm{s})$, além da razão entre os raios da voluta e da entrada do bocal $\left(r_{1} / r_{2}=1,2\right)$, descrito por Paltrinieri (2014). A sequência de cálculo é iniciada após uma estimativa inicial para a eficiência total-estática da turbina. Após a realização do procedimento de cálculo, uma nova eficiência é obtida e o processo se repete com essa nova eficiência até que a diferença entre a eficiência estimada e a calculada seja menor do que um valor admissível. Essa estimativa inicial da eficiência do estágio da turbina será de $75 \%$. 
Os princípios utilizados nessa metodologia obedecem às primeira e segunda leis da termodinâmica, além da equação da continuidade e a conservação do momento angular. A equação da continuidade é sempre utilizada em um plano normal à direção meridional do escoamento e é representada pela Equação (38), já considerando o fator de bloqueio $B K$, representando a redução na área efetiva do escoamento devido à camada limite desenvolvida nos componentes da turbina e seu valor sugerido é de 0,1 (MOUSTAPHA et al., 2003). Para os casos em que não há redução expressiva na área do escoamento, pode-se considerar $B K$ como nulo.

$$
\dot{m}=\rho A c_{m}(1-B K)
$$

Obedecendo a sequência descrita no artigo citado, o processo inicia-se com o cálculo do estado termodinâmico de estagnação no ponto 4 , a partir do estado termodinâmico de estagnação do ponto 1 e da hipótese de os processos entre a entrada do sistema até a entrada do rotor serem adiabáticos. Em seguida a sequência de cálculo respeita a seguinte ordem, obedecendo às leis governantes e aos dados de entrada do problema: componentes da velocidade nos pontos 4 e 5, segundo a geometria do triângulo de velocidades; estado termodinâmico estático em 4 , e estático e de estagnação em 5; dimensões do rotor; componentes de velocidade na raiz e ponta das pás em 5; e finalmente, as perdas no rotor. Importante observar que a pré-definição dos valores de entrada, como razão definida entre alguns raios é determinante para que os cálculos nessa etapa sejam realizados sem a necessidade de se realizar iterações. O procedimento de cálculo nos componentes do rotor é apresentado na Figura 5.

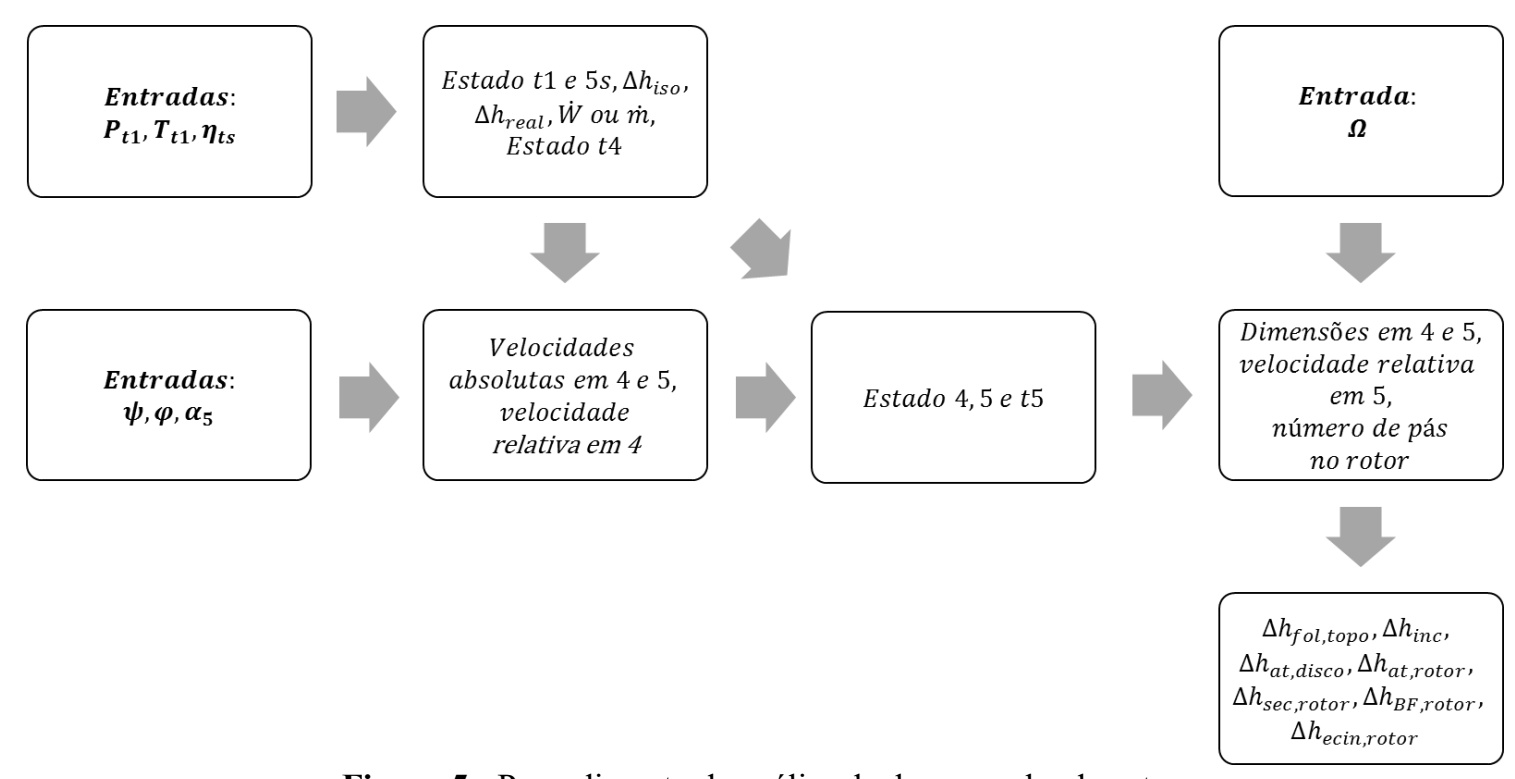

Figura 5 - Procedimento de análise de desempenho do rotor.

Definidas as dimensões do rotor, calcula-se o raio de saída do bocal, a partir da relação apresentada por Rahbar et al. (2014) e a componente tangencial da velocidade com base na conservação do momento angular. Então, um processo iterativo se faz necessário para o cálculo da componente meridional da velocidade em 3, visto que a entalpia estática depende da velocidade absoluta, que, por sua vez, depende das componentes tangencial e meridional. Por conta disso, estima-se um valor inicial para a densidade do fluido e o processo de cálculo é repetido até que se atinja a convergência. Consequentemente calcula-se os estados 
termodinâmicos estático e de estagnação na saída do bocal, conforme fluxograma mostrado na Figura 6.

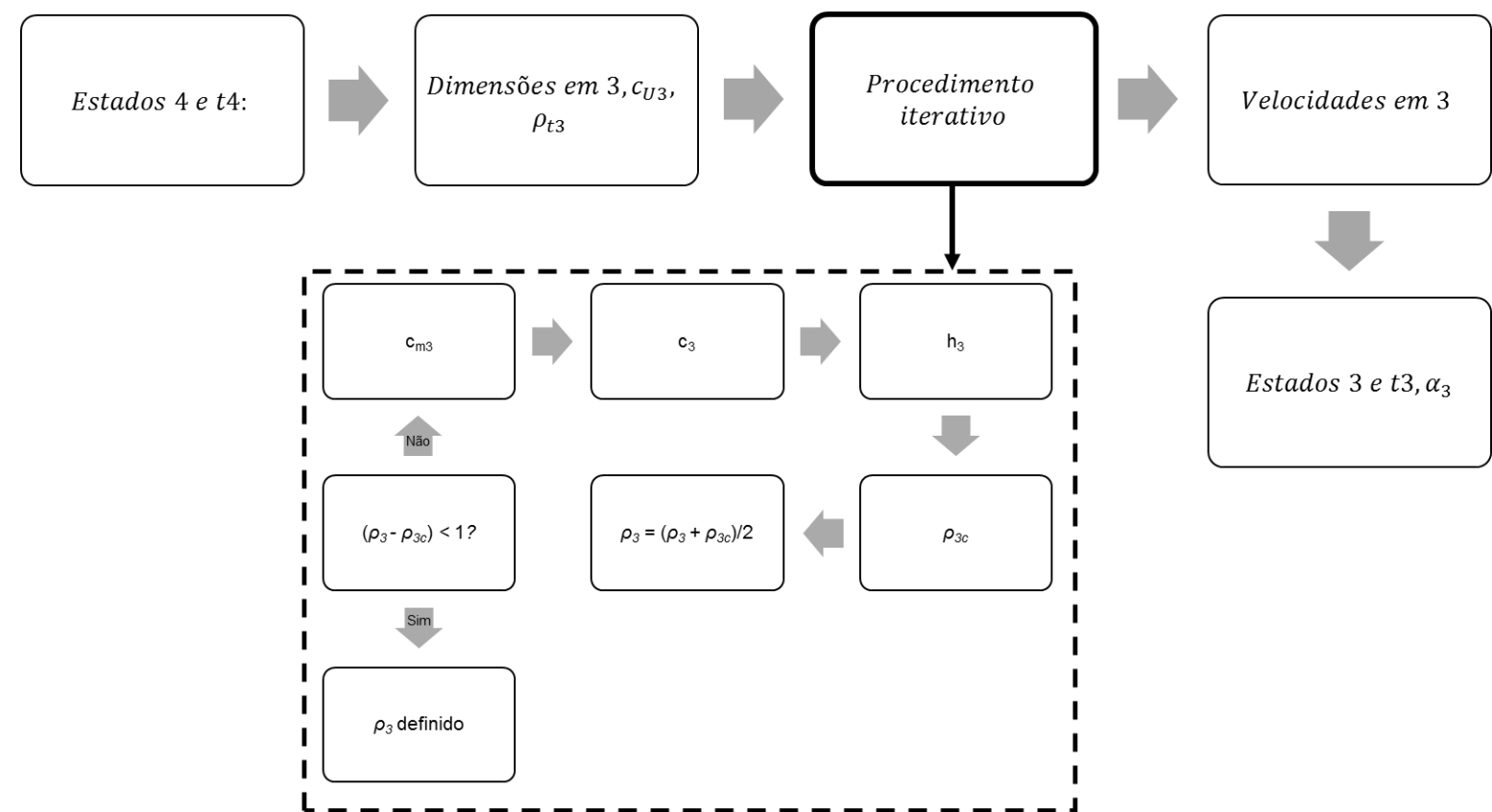

Figura 6 - Procedimento de análise de desempenho da saída do estator.

O cálculo do raio na entrada do bocal segue o mesmo procedimento realizado na saída desse componente e as componentes de velocidade são calculadas respeitando-se a conservação do momento angular, equação da continuidade e hipótese de processo adiabático no bocal, além de considerar que os ângulos das resultantes das velocidades em relação às componentes meridionais são iguais na entrada e na saída, para que as pás do bocal não possuam curvatura (PALTRINIERI, 2014). Em seguida calcula-se a perda ocorrida na voluta, cálculo dependente exclusivamente da velocidade na entrada do bocal e necessário para a definição do estado estático real em 2, visto que a partir desse cálculo é possível comparar as perdas ocorridas com o processo isentálpico. Então calcula-se os estados termodinâmicos estático e de estagnação nesse ponto bem como as perdas ocorridas no bocal, conforme caixas na parte de cima na Figura 6.

A modelagem da voluta é realizada com o cálculo do raio do ponto 1 a partir da razão definida na entrada do problema e, seguindo a Equação (1), calcula-se a velocidade na entrada desse componente. A partir desses últimos dados obtidos e da geometria apresentada na Figura 1, podem ser calculados o raio da seção transversal da voluta, bem como o estado termodinâmico estático em 1, conforme apresentado na Figura 7.

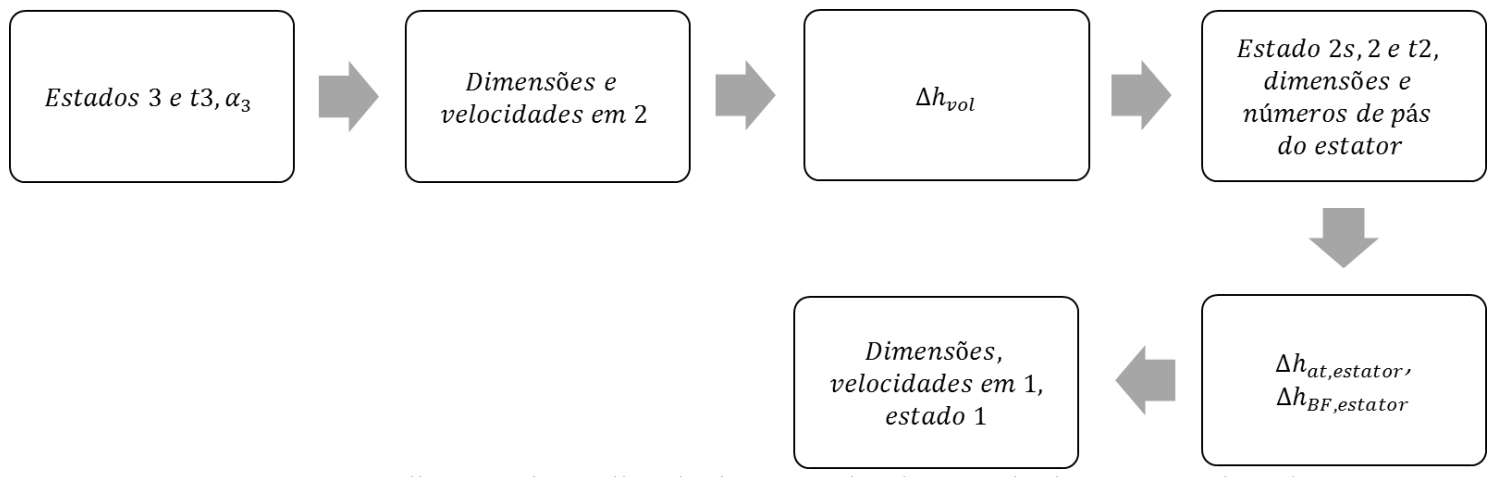

Figura 71 - Procedimento de análise de desempenho da entrada do estator e da voluta. 
Finalmente, calcula-se a eficiência corrigida da turbina com base na soma das perdas calculadas na modelagem de cada componente e o procedimento de cálculo é repetido, conforme já foi explicado anteriormente. Na Figura 8 é apresentado um fluxograma resumindo o procedimento de cálculo da turbina radial.

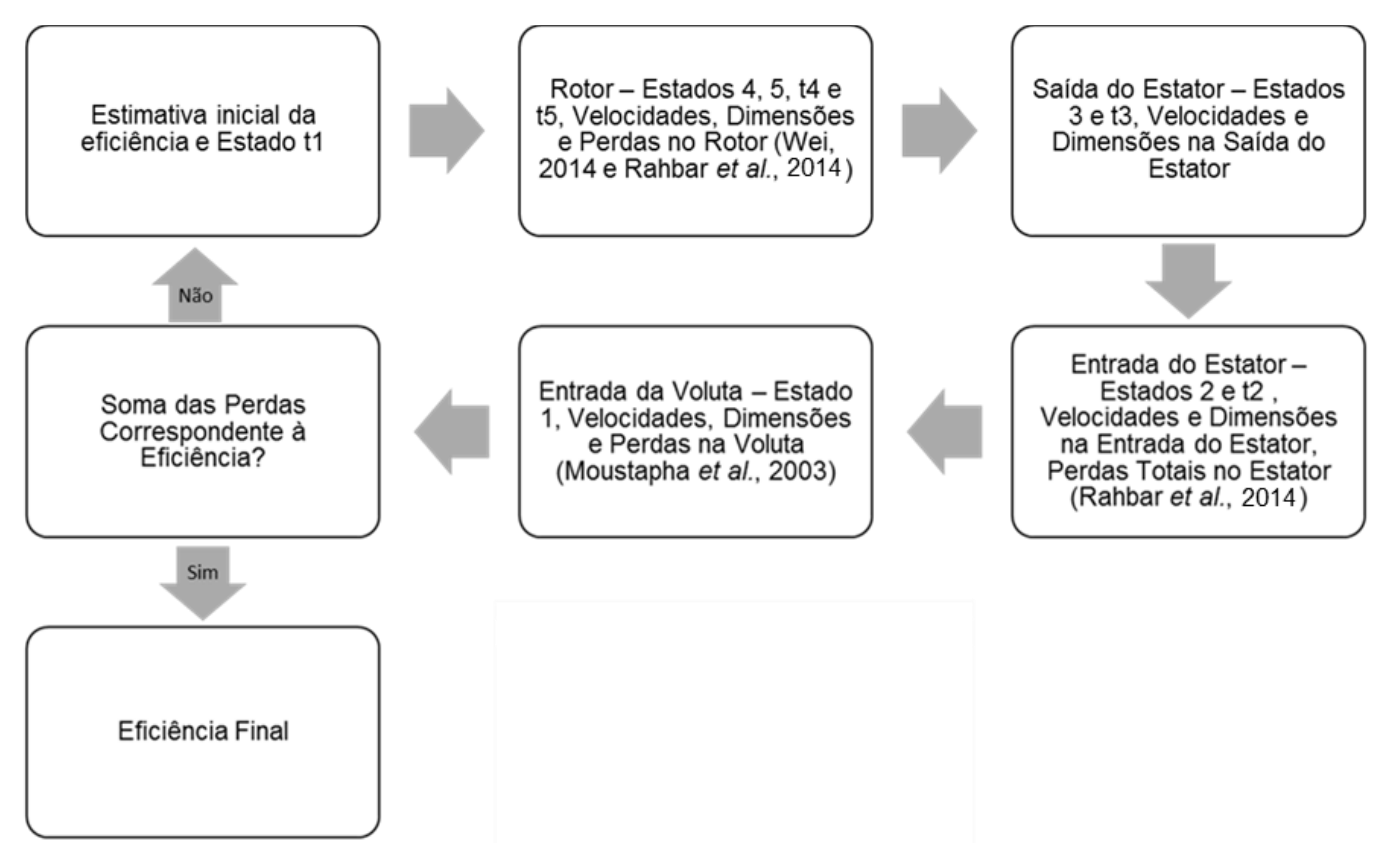

Figura 8 - Fluxograma do procedimento iterativo realizado.

Além disso, propõe-se a realização de procedimento de otimização da turbina em relação à eficiência do sistema utilizando-se a metodologia do enxame de partículas para que se encontre o ponto máximo global. Esse método bioinspirado baseia-se no comportamento de grupos de indivíduos de determinada espécie em busca de alimento, dentro do qual a maioria dos indivíduos segue aquele que conhece o melhor caminho até o alimento (ARORA, 2012). Esse método se caracteriza por uma geração de um primeiro conjunto de valores das variáveis de otimização, os indivíduos, e cálculo da função objetivo para cada um deles. O indivíduo que possuir o maior valor da função objetivo se torna o líder do conjunto e a continuidade do método se dá através da criação de uma nova geração de indivíduos, baseada no valor do líder do grupo e em coeficientes de geração aleatória. A cada nova geração, se o valor da função objetivo do líder for superado por algum outro indivíduo, este passa a ser o novo líder do grupo. O procedimento é realizado até que haja a convergência de valores da variável de otimização entre os demais indivíduos do grupo e o líder, dentro de um limite estabelecido.

No problema de otimização da turbina, as variáveis de otimização são os valores de entrada da metodologia de cálculo, cujos intervalos estão expressos anteriormente, e a função objetivo é o produto entre a eficiência da turbina e a eficiência do ciclo termodinâmico (ambos os valores serão expressos em porcentagem), sendo definido um número máximo de 1000 iterações em cada processo. A intenção nessa fórmula da função objetivo é promover o melhor balanço entre a eficiência da turbina e a eficiência do ciclo termodinâmico.

As simulações foram realizadas em um computador com processador Intel (R) Core (TM) i7-3632QM de quatro núcleos de 2,20 GHz com 8 GB de memória RAM. 


\section{Resultados da otimização}

Seguindo a metodologia descrita na seção anterior, realizou-se um estudo de otimização para o projeto de uma turbina radial de potência de $10 \mathrm{~kW}$, comparando-se os resultados dos quatro fluidos descritos na Tabela 1 , assim como as variáveis de entrada já otimizadas. $\mathrm{O}$ tempo de processamento da otimização para cada fluido foi de aproximadamente 12 minutos.

Tabela 1 - Variáveis de entrada otimizadas utilizando diferentes fluidos para o cálculo da turbina.

\begin{tabular}{|c|c|c|c|c|c|}
\hline Fluido & R227ea & R245fa & R123 & R236fa & R236ea \\
\hline$T_{t 1}(K)$ & 424,2 & 409,3 & 499,7 & 414,1 & 430,5 \\
\hline$P_{t 1}(k P a)$ & 3021,6 & 1352,1 & 1742,1 & 1838,6 & 1816,1 \\
\hline$E R_{t s}(-)$ & 2,896 & 2,751 & 3,213 & 3,217 & 3,053 \\
\hline$\Omega(r p m)$ & 78977 & 72879 & 79309 & 62902 & 77845 \\
\hline$\psi(-)$ & 0,800 & 0,801 & 0,818 & 0,816 & 0,827 \\
\hline$\varphi(-)$ & 0,261 & 0,337 & 0,267 & 0,212 & 0,254 \\
\hline
\end{tabular}

Seguindo o comparativo proposto, os resultados da função objetivo estão apresentados na Tabela 2. O fluido R123 apresentou o melhor produto entre as eficiências do ciclo termodinâmico e de turbina, seguido pelo R236ea. A Figura 9 e Figura 10 representam essa análise, sendo o R123 o fluido com maior combinação entre eficiência de turbina e eficiência de ciclo termodinâmico. Segundo a Tabela 3, o fluido R245fa apresentou o melhor desempenho de turbina, seguido pelo R227ea. Porém, esses dois fluidos apresentaram um desempenho de ciclo muito abaixo (1\%) do melhor desempenho, o R123, que por sua vez, apresenta uma eficiência de turbina pouco menos de $2 \%$ menor do que o R245fa. Em termos de ordem de grandeza da eficiência de turbina (da ordem de $80 \%$ ), $2 \%$ a menos de eficiência, porém com $1 \%$ de ganho na eficiência de ciclo (grandeza na ordem de $8 \%$ ) representa um maior benefício para o R123, que além de tudo, apresenta uma vazão mássica menor, demandando dessa forma, uma menor quantidade de fluido para sua operação. É importante observar que as dimensões da turbina respeitam dimensões de ordem de grandeza maiores do que $1 \mathrm{~mm}$, possibilitando sua manufaturabilidade. Além disso, o fluido R123 é o que apresenta em geral, o segundo sistema mais compacto, o que representa uma melhor relação custo-benefício pela quantidade de material utilizado na fabricação da turbina.

As eficiências de turbina apresentadas pelo resultado da otimização estão na mesma ordem de grandeza do que as apresentadas na metodologia de cálculo de perdas e otimização de Rahbar et al. (2014), em torno de 75\%. Importante ressaltar que os valores do coeficiente de carga dos fluidos analisados mantiveram-se bastante próximos de 0,815 , indicando um indício de proximidade do ponto ótimo nesse valor para essa grandeza. Nos resultados analisados na literatura, o aumento desse coeficiente provocou queda na eficiência de turbina. 


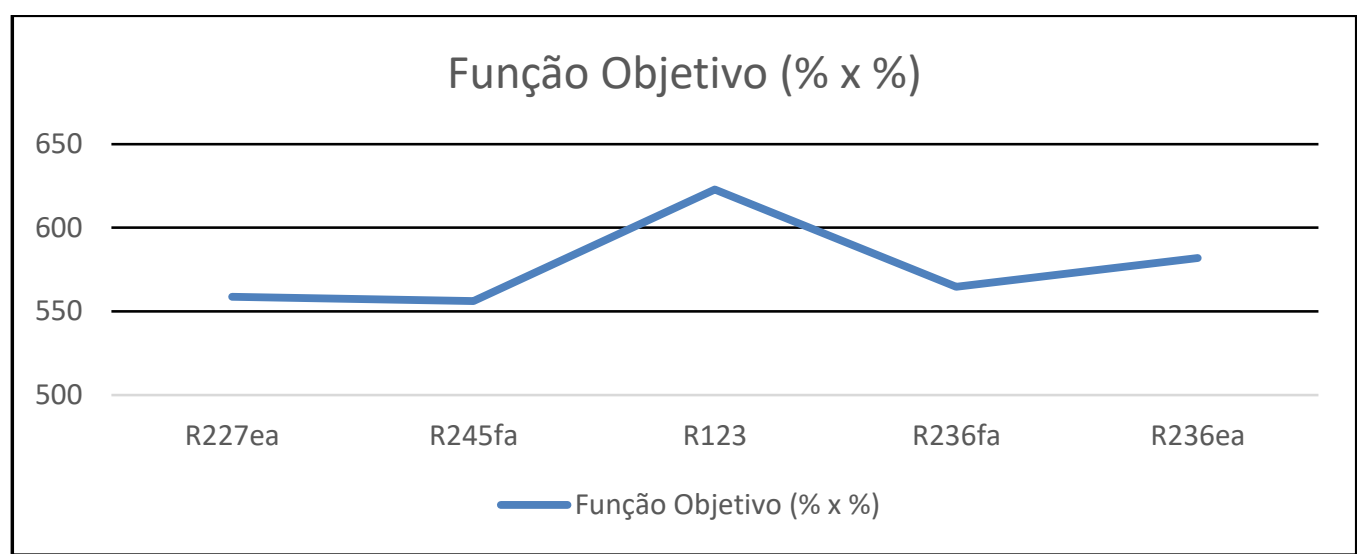

Figura 9- Gráfico do valor da função objetivo (\% x \%) versus fluido de trabalho no processo de otimização.

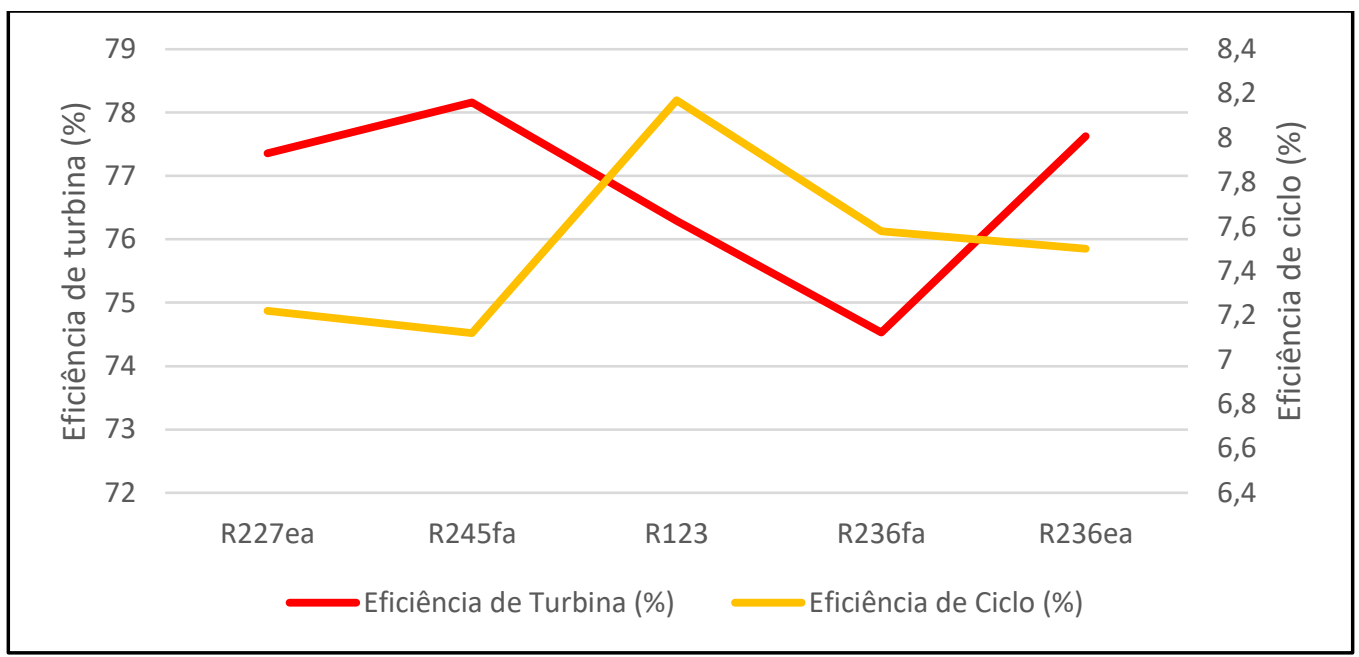

Figura 10 - Gráfico da eficiência de turbina (\%) e da eficiência de ciclo (\%) versus fluido de trabalho no processo de otimização.

Tabela 2 - Valores da função objetivo otimizada.

\begin{tabular}{|c|c|}
\hline Fluido & $\eta_{t s}(\%) \cdot \eta_{t, \text { ciclo }}(\%)$ \\
\hline R227ea & 558,7 \\
\hline R245fa & 556,2 \\
\hline R123 & 622,9 \\
\hline R236fa & 564,7 \\
\hline R236ea & 581,9 \\
\hline
\end{tabular}


Tabela 3 - Resultados do processo de otimização

\begin{tabular}{|c|c|c|c|c|c|}
\hline Fluido & R227ea & R245fa & $\mathbf{R 1 2 3}$ & R236fa & R236ea \\
\hline$\eta_{\text {ts }}(\%)$ & 77,36 & 78,16 & 76,29 & 74,53 & 77,63 \\
\hline$\eta_{t, \text { ciclo }}(\%)$ & 7,22 & 7,12 & 8,17 & 7,58 & 7,50 \\
\hline$\dot{m}(\mathrm{~kg} / \mathrm{s})$ & 0,806 & 0,636 & 0,513 & 0,664 & 0,626 \\
\hline$\alpha_{4}\left({ }^{\circ}\right)$ & 71,92 & 67,19 & 71,92 & 75,44 & 72,96 \\
\hline$U_{4}(\mathrm{~m} / \mathrm{s})$ & 129,7 & 145,9 & 160,7 & 141,5 & 144,7 \\
\hline$c_{4}(\mathrm{~m} / \mathrm{s})$ & 109,2 & 126,8 & 138,4 & 119,2 & 125,3 \\
\hline$c_{5}(\mathrm{~m} / \mathrm{s})$ & 33,9 & 49,2 & 43,0 & 30,0 & 36,7 \\
\hline$w_{4}(\mathrm{~m} / \mathrm{s})$ & 42,6 & 57,1 & 51,9 & 39,7 & 44,4 \\
\hline$w_{5 \text { topo }}(\mathrm{m} / \mathrm{s})$ & 106,4 & 123,3 & 125,5 & 115,3 & 126,5 \\
\hline$d_{\text {max }}(\mathrm{mm})$ & 58,35 & 75,05 & 70,56 & 77,06 & 67,09 \\
\hline$d_{1}(\mathrm{~mm})$ & 49,19 & 61,65 & 60,04 & 66,17 & 56,17 \\
\hline$d_{4}(\mathrm{~mm})$ & 31,36 & 38,23 & 38,71 & 42,96 & 35,51 \\
\hline$d_{5 \text { tip }}(\mathrm{mm})$ & 24,39 & 29,63 & 28,40 & 33,80 & 29,71 \\
\hline$b_{4}(\mathrm{~mm})$ & 2,25 & 2,96 & 2,41 & 2,97 & 2,98 \\
\hline$Z_{\text {rotor }}(-)$ & 12 & 11 & 12 & 14 & 13 \\
\hline
\end{tabular}

$\mathrm{Na}$ Tabela 4, apresenta-se a contribuição percentual dos mecanismos de perda nas turbinas otimizadas para cada fluido. Dadas as dimensões reduzidas, observou-se que as perdas no bordo de fuga das pás do estator e do rotor podem ser consideradas desprezíveis, bem como as perdas por incidência, estas minimizadas a partir da escolha do ângulo de entrada do fluido no rotor sendo igual ao ângulo ótimo, opção possibilitada pela menor temperatura de operação nesse tipo de sistema, conforme já discutido nas seções anteriores. Nota-se também pequenas dimensões do sistema acarreta em um aumento na dimensão relativa entre a área de escoamento, e a dimensão dos componentes. Dessa forma, as perdas por atrito no estator e na folga de topo das pás do rotor são os mecanismos responsábeis por mais de $50 \%$ das perdas totais em cada turbina. A maior velocidade relativa na linha média provocou a maior taxa de perdas por escoamentos secundários na turbina operando com R245fa. 
Tabela 4 - Contribuição de cada mecanismo de perda em cada turbina otimizada.

\begin{tabular}{|c|c|c|c|c|c|}
\hline Fluido & R227ea & R245fa & $\mathbf{R 1 2 3}$ & R236fa & R236ea \\
\hline$\Delta h_{\text {vol }}(\%)$ & 8,73 & 9,06 & 8,58 & 7,66 & 8,90 \\
\hline$\Delta h_{B F, \text { stator }}(\%)$ & 0,04 & 0,03 & 0,02 & 0,05 & 0,06 \\
\hline$\Delta h_{\text {at,estator }}(\%)$ & 30,88 & 25,15 & 35,04 & 34,22 & 29,43 \\
\hline$\Delta h_{\text {fol,topo }}(\%)$ & 28,59 & 20,60 & 25,95 & 35,41 & 32,00 \\
\hline$\Delta h_{\text {at,rotor }}(\%)$ & 8,82 & 10,95 & 8,51 & 6,85 & 8,14 \\
\hline$\Delta h_{\text {sec,rotor }}(\%)$ & 6,98 & 7,82 & 6,58 & 6,21 & 6,78 \\
\hline$\Delta h_{\text {ecin,rotor }}(\%)$ & 14,58 & 25,38 & 14,04 & 8,04 & 13,49 \\
\hline$\Delta h_{B F, \text {,otor }}(\%)$ & 0,08 & 0,05 & 0,04 & 0,10 & 0,11 \\
\hline$\Delta h_{\text {inc }}(\%)$ & 0,27 & 0,09 & 0,06 & 0,32 & 0,09 \\
\hline$\Delta h_{\text {at, } \text {,isco }}(\%)$ & 1,04 & 0,89 & 1,18 & 1,14 & 0,99 \\
\hline
\end{tabular}

\section{Conclusão}

Conclui-se que a metodologia adotada apresenta resultados de eficiência de turbina bastante satisfatórios, com valores acima dos $70 \%$ de eficiência com um bom balanço entre eficiência de turbina e eficiência de ciclo, dentro das limitações impostas dentro do uso de fontes de energia de baixas temperaturas. Dentro dos parâmetros da comparação, o fluido R123 foi o que apresentou melhor desempenho no balanço entre eficiência de ciclo e eficiência de turbina, bem como uma relação custo-benefício se comparar as dimensões da turbina e quantidade de fluido atuando no sistema. Além disso, da análise das contribuições de cada tipo de perda e suas contribuições sobre a perda total nas turbinas, pode-se realizar um comparativo satisfatório entre as hipóteses realizadas sobre o conceito físico dos mecanismos de perdas e seu resultado matemático, apresentando evidências de que os modelos utilizados correspondem aos resultados práticos em um possível experimento.

\section{Referências}

ARORA, J. S. Introduction to optimum design. 3. ed. Waltham: Elsevier, 2012.

ÇENGEL, Y. A.; BOLES, M. A. Termodinâmica. 7. ed. Porto Alegre: AMGH Editora Ltda., 2013.

ÇENGEL, Y. A.; CIMBALA, J. M. Mecânica dos fluidos: fundamentos e aplicações. 3. ed. Porto Alegre: AMGH Editora Ltda., 2015.

DIXON, S. L.; HALL, C. A. Fluid mechanics and thermodynamics of turbomachinery. 6. ed. Burlington: Butterworth Heinemann, 2010.

GLASSMAN, A. J. Computer program for design analysis of radial-inflow turbines. NASA Technical Note, TN D-8164, Lewis Research Center, Cleveland, Ohio, 1976. 
GLASSMAN, A. J. Enhanced analysis and user's manual for radial-inflow turbine conceptual design code RTD. Toledo: University of Toledo, 1995.

MANTEGAZZA, P.; CABEZAS-GÓMEZ, L.; MAGAZONI, F. C. Modelagem preliminar de turbina radial com entrada radial com o método da linha média. ENCONTRO REGIONAL DE MATEMÁTICA APLICADA E COMPUTACIONAL, 2017, Bauru. Caderno de trabalhos completos e resumos. Bauru: Unesp, Faculdade de Ciências, 2017. p. 200 - 207.

MOUSTAPHA, H. et al. Axial and radial turbines. White River Junction: Concepts NREC, 2003.

PALTRINIERI, A. A mean-line model to predict the design performance of radial inflow turbines in organic rankine cycles. 2014. 145 f. Tesi (Laurea Magistrale in Ingegneria Energetica) - Dipartimento di Ingegneri Industriale, Università Degli Studi di Padova/Institut für Energietechnik, Technische Universität Berlin, Padova/Berlim, 2014.

RAHBAR, K. et al. Modelling and optimization of organic rankine cycle based on a smallscale radial inflow turbine. Energy Conversion and Management, v. 91, p. 186-198, dez. 2014.

VENTURA, C. A. M. et al. Preliminary design and performance estimation of radial inflow turbines: an automated approach. Journal of Fluids Engineering, v. 134, n. 3, p. 1-13, mar. 2012.

WEI, Z. Meanline analysis of radial inflow turbines at design and off-design conditions. 2014. 157 f. Thesis (Master of Applied Science in Aerospace Engineering) - Faculty of Graduate and Postdoctoral Affairs, Carleton University, Ottawa, 2014.

WHITFIELD, A.; BAINES, N.C. Design of radial turbomachines. London: Longman Scientific \& Technical, 1991.

Artigo recebido em jun. 2017 e aceito em nov. 2017. 